\title{
On the Kennicutt-Schmidt scaling law of submillimetre galaxies
}

\author{
O. Miettinen ${ }^{1}$, I. Delvecchio ${ }^{1}$, V. Smolčić ${ }^{1}$, M. Aravena ${ }^{2}$, D. Brisbin ${ }^{2}$, and A. Karim ${ }^{3}$ \\ ${ }^{1}$ Department of Physics, Faculty of Science, University of Zagreb, Bijenička cesta 32, 10000 Zagreb, Croatia \\ e-mail: oskari@phy.hr \\ 2 Núcleo de Astronomía, Facultad de Ingeniería, Universidad Diego Portales, Av. Ejército 441, Santiago, Chile \\ 3 Argelander-Institut für Astronomie, Universität Bonn, Auf dem Hügel 71, 53121 Bonn, Germany
}

Received 11 May 2017 / Accepted 30 May 2017

\begin{abstract}
Context. The star formation rate (SFR) per unit area correlates well with the gas surface density for different types of galaxies. However, this Kennicutt-Schmidt (K-S) law has not yet been examined for a large, homogeneously selected sample of submillimetre galaxies (SMGs), which could provide useful SF implementation information for models of massive galaxy formation and evolution. Aims. We aim at determining the K-S law parameters for the first time for a well-selected, statistical sample of SMGs.

Methods. We used the Atacama Large Millimetre/submillimetre Array (ALMA) to conduct a high resolution $\left(0^{\prime \prime} \cdot 2\right), 870 \mu \mathrm{m}$ continuum imaging survey of 40 SMGs, which were initially selected at $1.1 \mathrm{~mm}$ in the COSMOS field. We analysed a sample of 32 out of the 40 target SMGs, for which our new ALMA $870 \mu \mathrm{m}$ data provide information about the spatial extent of dust emission, and all of which have dust-obscured SFR and dust-based gas mass estimates available from our previous study.

Results. We divided our sample into equally large subsamples of main-sequence (MS) objects and starbursts (factor of $>3$ above the MS), and found their K-S relations to be of the form $\Sigma_{\mathrm{SFR}} \propto \Sigma_{\text {gas }}^{0.81 \pm 0.01}$ and $\Sigma_{\mathrm{SFR}} \propto \Sigma_{\text {gas }}^{0.84 \pm 0.39}$, respectively.

Conclusions. The slightly sub-linear K-S slopes we derived suggest that the SF efficiency (SFE) is nearly constant across the $\Sigma_{\text {gas }}$ range probed. Under the assumption of a Galactic CO-to- $\mathrm{H}_{2}$ conversion factor $\left(\alpha_{\mathrm{CO}}\right)$ for the whole sample, the MS SMGs obey a constant global SFE of about 21\% per 100 Myr, while that of starburst SMGs is about 27\% per 100 Myr. The corresponding gas depletion times are $\sim 480 \mathrm{Myr}$ and $370 \mathrm{Myr}$. On average, our SMGs have $\Sigma_{\text {gas }} \gtrsim 10^{3.9} M_{\odot} \mathrm{pc}^{-2}$, which suggests that they are Eddingtonlimited. This is consistent with the theoretical expectation of a linear K-S relation for such systems. However, size measurements of the CO-emitting regions of SMGs, and the $\alpha_{\mathrm{CO}}$ values of SMGs are needed to further constrain their $\Sigma_{\text {gas }}$ values.
\end{abstract}

Key words. galaxies: evolution - galaxies: formation - galaxies: starburst - galaxies: star formation - submillimeter: galaxies

\section{Introduction}

The empirical Kennicutt-Schmidt (K-S) law quantifies the amount of cold interstellar gas required to sustain a given star formation rate (SFR; Schmidt 1959; Kennicutt 1998, hereafter K98; see Kennicutt \& Evans 2012, for a review). Specifically, K98 found that the galaxy-integrated SFR surface density $\left(\Sigma_{\text {SFR }}\right)$ and total (atomic plus molecular, $\mathrm{HI}+\mathrm{H}_{2}$ ) gas surface density $\left(\Sigma_{\text {gas }}\right)$ of normal star-forming disk galaxies and luminous infrared (IR) selected starbursts are tightly linked to each other over about five decades in $\Sigma_{\text {gas }}$ through a functional form of $\Sigma_{\mathrm{SFR}} \propto \Sigma_{\text {gas }}^{1.4 \pm 0.15}$. Besides normal spirals and starbursts studied by K98, the K-S-type star-formation relations have been explored for different types of galaxies with different physical properties, such as low-surface-brightness galaxies (Wyder et al. 2009) and luminous IR galaxies (García-Burillo et al. 2012). However, studies of the K-S law of the most intensely star-forming dusty galaxies, the so-called submillimetre galaxies (SMGs; see Casey et al. 2014, for a review), are not only few in number, but they have also been based on small, heterogenous, and partly overlapping literature samples (Bouché et al. 2007; Daddi et al. 2010b, hereafter D10b; Bothwell et al. 2010; Genzel et al. 2010; see also Hodge et al. 2015). For instance, D10b found that while SMGs and normal disks have a common K-S slope of 1.42, which is fully consistent with the K98 value, the SMGs occupy a higher $\Sigma_{\mathrm{SFR}}$ regime of the K-S diagram with 0.9 dex higher normalisation. This is considered an indication that SMGs, which are potentially driven by gas-rich mergers, are relatively more efficient star formers (see also Genzel et al. 2010, 2015).

Inherently, the observed galactic scale $\mathrm{K}-\mathrm{S}$ relation is a manifestation of the low global SF efficiency (SFE). Although the exact parameters of the K-S relation are dependent on several factors (e.g. the SFR and gas tracers used; e.g. Krumholz \& Thompson 2007; Liu et al. 2011; Momose et al. 2013), the global SFE appears to be only a few percent (e.g. K98; Bigiel et al. 2008; Genzel et al. 2010). In this regard, to better understand the overall role played by SMGs in the formation and evolution of massive galaxies, it is pivotal to try to quantify how efficiently SMGs turn their gas into stars, yet this requires an analysis of a well-selected statistical source sample.

In this Letter, we report our results regarding the K-S law of SMGs, which were detected at $870 \mu \mathrm{m}$ with the Atacama Large Millimetre/submillimetre Array (ALMA). This represents the first homogenous, statistically more significant sample of SMGs for which the K-S law has been explored so far. The SMG sample and observations are described in Sect. 2, while the analysis and results are described and discussed in Sect. 3. Section 4 summarises our results. Throughout this Letter, we adopt a Chabrier (2003) initial mass function (IMF), and assume 
a $\Lambda$ CDM (Lambda cold dark matter) cosmology with the dark energy density $\Omega_{\Lambda}=0.70$, and total matter density $\Omega_{\mathrm{m}}=0.30$, while the Hubble constant is set at $H_{0}=70 \mathrm{~km} \mathrm{~s}^{-1} \mathrm{Mpc}^{-1}$.

\section{Source sample and ALMA observations}

The target SMGs, called AzTEC/C1-C27, were originally uncovered by the AzTEC $\lambda_{\text {obs }}=1.1 \mathrm{~mm}$ blank-field continuum survey of the inner $0.72 \mathrm{deg}^{2}$ of the COSMOS field (Aretxaga et al. 2011). The sources AzTEC/C1-C27 correspond to a signal-to-noise limited subsample of the AzTEC single-dish sources with $S / N_{1.1 \mathrm{~mm}}^{\mathrm{AzTEC}} \geq 5.5\left(S_{1.1 \mathrm{~mm}}=5.7-13 \mathrm{mJy}\right)$, and were observed as part of our ALMA follow-up survey in Cycle 2 at $\lambda_{\text {obs }}=1.3 \mathrm{~mm}$ and $\sim 1^{\prime \prime} .6 \times 0$ "'.9 resolution (PI: M. Aravena; Aravena et al., in prep.). The dedicated ALMA pointings towards these 27 AzTEC sources revealed 41 sources altogether, at a $S / N_{1.3 \mathrm{~mm}}^{\mathrm{ALMA}} \geq 5\left(S_{1.3 \mathrm{~mm}}=0.55-7.25 \mathrm{mJy}\right)$.

We followed up the $1.3 \mathrm{~mm}$ sources detected towards AzTEC/C1-C27 with ALMA in Cycle 4 using Band 7 continuum observations at $\lambda_{\mathrm{obs}}=870 \mu \mathrm{m}$ under project 2016.1.00478.S (PI: O. Miettinen). The observations were carried out on 28 October 2016. Altogether, 40 ALMA $1.3 \mathrm{~mm}$ sources were covered by 34 pointings (16!'7 FWHM field-ofview), with a total on-source integration time of about $1.3 \mathrm{~min}$ per pointing (AzTEC/C $3 b$ was not observed). The observations were made using the $12 \mathrm{~m}$ array with 41 antennas, where the baselines ranged from $18.6 \mathrm{~m}(21.3 \mathrm{k} \lambda)$ to $1.1 \mathrm{~km}(1260 \mathrm{k} \lambda)$. The large number of antennas allowed us to reach an excellent $u v$ coverage even in the aforementioned short integration time. The amount of precipitable water vapour was only about $0.38 \mathrm{~mm}$. The phases were calibrated by observations of the Seyfert 1 galaxy J0948+0022, while the BL Lac object J1058+0133 was observed for amplitude and bandpass calibration. The correlator was configured in four spectral windows centred at $336.5 \mathrm{GHz}$ and $338.5 \mathrm{GHz}$ in the lower sideband, and at $348.5 \mathrm{GHz}$ and $350.5 \mathrm{GHz}$ in the upper sideband, each covering a bandwidth of $1.875 \mathrm{GHz}$ divided into 128 channels of $15.625 \mathrm{MHz}$ (with dual polarisation). Hence, the total bandwidth available for continuum observations was $7.5 \mathrm{GHz}$.

The visibility data were edited, calibrated, and imaged using the standard ALMA pipeline of the Common Astronomy Software Applications (CASA; McMullin et al. 2007) version 4.7.0. The final images were created using the tclean task by adopting Briggs weighting with a robust parameter of 0.5. The resulting images have a typical (median) synthesised beam of $0^{\prime \prime}$. $192 \times 00^{\prime \prime} 176$, while the typical $1 \sigma \mathrm{rms}$ noise of the final images is $0.155 \mathrm{mJy}^{\text {beam }}{ }^{-1}$, which was estimated from emission-free regions after correction for the primary beam (PB) response.

Out of the 40 target sources, 36 were detected with a $\mathrm{S} / \mathrm{N}$ ratio ranging from 5.9 to 33 (see Fig. A.1). The four sources that were not detected are AzTEC/C1b, C8b, C10c, and C13b $\left(S / N_{1.3 \mathrm{~mm}}^{\mathrm{ALMA}}=5.2,5.5,5.1\right.$, and 10.2, respectively $)$. A potential reason for these non-detections is that the emission was resolved out at 0 '!2 resolution. To test this possibility, we convolved the images with a Gaussian smoothing kernel of different radii. No emission was recovered towards AzTEC/C1b ( 5".6 south-west (SW) of the phase centre (PC)) and C10c (source at the PC), which suggests that these sources might be spurious. Indeed, AzTEC/C1b and C10c have no multiwavelength counterparts, unlike C8b and C13b (Brisbin et al. 2017). Also, the map smoothing did not reveal any clear source at the $1.3 \mathrm{~mm}$ position of AzTEC/C13b, and in this case the non-detection might be caused by PB attenuation, because the source lies $\sim 6$ ". 4 to the $\mathrm{SW}$ of the PC, where the map starts to become noisy. However, although AzTEC/C8b also lies near the noisy map edge $\left(\sim 7^{\prime \prime}\right.$ to the SW from the PC), the source appeared in smoothed images (starting to become visible at $00^{\prime \prime} 30 \times 0, \prime 25$ resolution, where the corresponding map rms noise is $\sim 0.2 \mathrm{mJy}^{b^{-1}} \mathrm{mem}^{-1}$ ) with a hint of two components of $5 \sigma$ and $4.7 \sigma$ significance separated by 0 '?26. AzTEC/C8b also has a large radio-emitting full width at half maximum $(\mathrm{FWHM})$ size of 1 !'7 $\times 1$ !'1 (Miettinen et al. 2017a, hereafter M17a), which is consistent with the finding that its dust-emitting region was resolved out. Owing to the location of $\mathrm{C} 8 \mathrm{~b}$ near the noisy map boundary, and the fact that it was resolved out at $0^{\prime \prime} .2$ resolution, we do not consider it in the subsequent analysis to preserve the homogeneity of the data set.

\section{Data analysis, results, and discussion}

An integral part of the present analysis is to determine the spatial scale of the observed-frame $870 \mu \mathrm{m}$ emission. For this purpose, we used the NRAO Astronomical Image Processing System (AIPS) software package. Specifically, the beam-deconvolved (intrinsic) sizes were derived through two-dimensional elliptical Gaussian fits to the image plane data using the AIPS task JMFIT. The Gaussian fitting was performed inside a rectangular box enclosing the source, and the fit was restricted to the pixel values of $\geq 2.5 \sigma$.

In the subsequent analysis, we use the deconvolved major axis FWHM as the diameter of the source, because the major axis represents the physical source extent in the case of isotropically oriented disks. All the sources were resolved along the major axis; the deconvolved FWHM was always found to be larger than one-half the synthesised beam major axis FWHM (see Table A.1). The median value of $\mathrm{FWHM}_{\text {maj }}$ is $0^{\prime \prime} 31_{-0.10}^{+0.15}$ $\left(2.4_{-0.8}^{+1.1} \mathrm{kpc}\right)$, where the uncertainty represents the 16 th-84th percentile range. This is in good agreement with previous studies of SMG sizes measured through ALMA $870 \mu \mathrm{m}$ observations (Simpson et al. 2015; Hodge et al. 2016), although the source is not always well modelled with an elliptical Gaussian profile (Fig. A.1). As a consistency check, we also used CASA to determine the source sizes (the imfit task), and found very good agreement with our AIPS/JMFIT results, the mean (median) ratio between the two being $\langle\operatorname{Size}(\mathrm{AIPS}) / \operatorname{Size}(\mathrm{CASA})\rangle=1.06$ (1.02).

The source radius, which enters into the calculation of the surface densities, was defined as $R=0.5 \times F W H M_{\text {maj }}$, which is appropriate for a circular disk. Both the SFR and gas mass $\left(M_{\text {gas }}\right)$ values were adopted from Miettinen et al. (2017b, hereafter M17b), who used the latest version of MAGPHYS (da Cunha et al. 2015) to fit the panchromatic spectral energy distributions (SEDs) of the target SMGs. The number of SMGs that have both the SED and size information available is 32 , and their redshifts range from $z=1.1_{-1.1}^{+2.6}$ to $z=5.3_{-1.1}^{+0.7}(40.6 \%$ are spectroscopically confirmed, while the remaining redshifts are photometric; Brisbin et al. 2017).

The best-fit MAGPHYS SEDs were integrated over the restframe wavelength range of $\lambda_{\text {rest }}=8-1000 \mu \mathrm{m}$ to derive the IR luminosities $\left(L_{\mathrm{IR}}\right)$. The values of $L_{\mathrm{IR}}$ were then used to estimate the dust-obscured, 100 Myr averaged SFR using the K98 relationship.

The gas masses were estimated using the Scoville et al. (2016) calibration and employing the ALMA $1.3 \mathrm{~mm}$ flux densities of the sources. These dust-based $M_{\text {gas }}$ values refer to the molecular $\left(\mathrm{H}_{2}\right)$ gas mass (see M17b for further details). We note that similar to the canonical K-S relation (K98), which assumes a Galactic CO-to- $\mathrm{H}_{2}$ conversion factor $\left(\alpha_{\mathrm{CO}}\right)$ for both the normal disks and starbursts, the Scoville et al. (2016) 

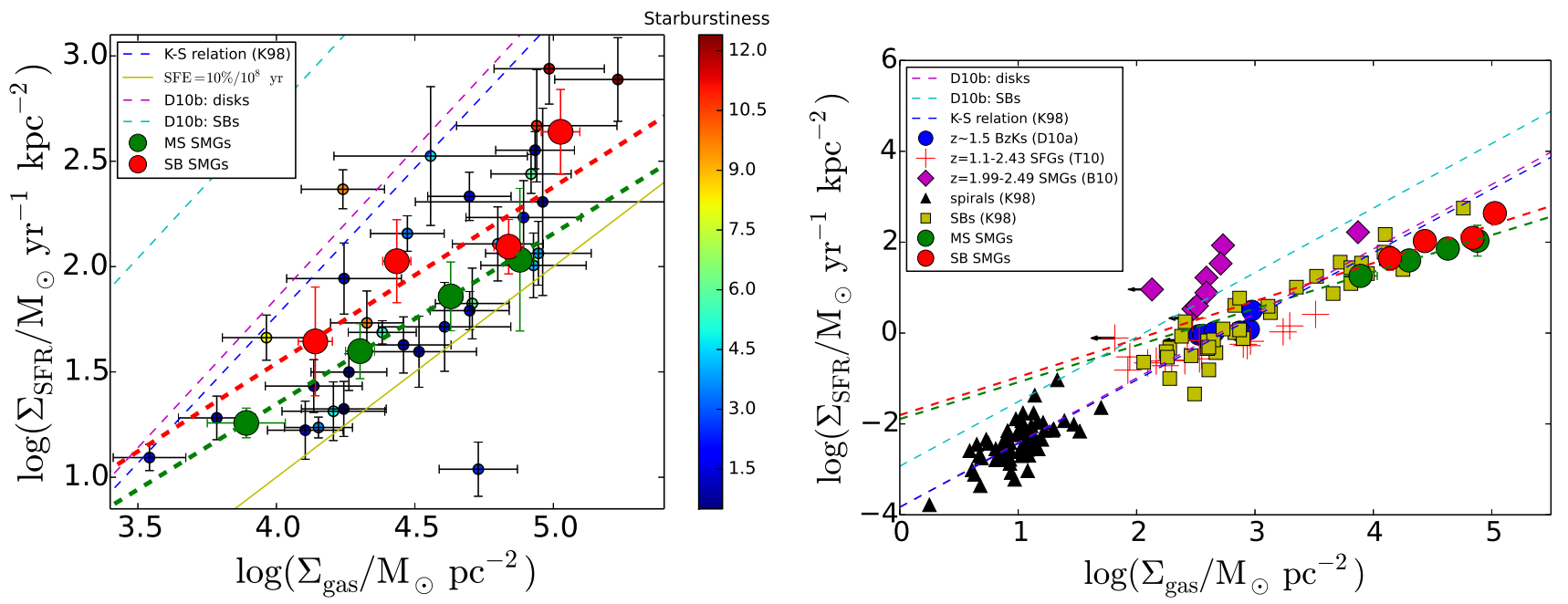

Fig. 1. Left: Kennicutt-Schmidt diagram for the target SMGs. The individual data points are colour-coded with the distance from the Speagle et al. (2014) MS as shown in the colour-bar on the right. The green and red filled circles represent the mean values of the binned MS and starburst data, where the latter population is defined as lying above the MS by a factor of $>3$. Each bin contains four SMGs, and the error bars represent the standard errors of the mean values (see Table A.2). The green and red dashed lines represent the least squares fits to the binned data sets, the blue dashed line shows the K98 relationship, and the magenta and cyan dashed lines show the D10b relations for disks and starbursts, respectively. For reference, the yellow solid line corresponds to a constant global SFE of $10 \%$ per $100 \mathrm{Myr}$, which corresponds to a gas depletion time of $\tau_{\text {dep }}=1$ Gyr. Right: binned averages from the left panel compared with selected literature studies. The black triangles and yellow squares show the spiral galaxy and starburst data from K98, respectively, the red plus signs show the $z=1.10-2.43$ star-forming galaxy data from Tacconi et al. (2010), the blue circles show the $z=1.414-1.6$ BzK-selected disk galaxies from Daddi et al. (2010a), and the magenta diamonds represent the $z=1.21-2.49$ SMG data from Bothwell et al. (2010). The arrows pointing left indicate upper limits to $\Sigma_{\text {gas }}$. The dashed lines have the same meaning as in the left panel.

method is calibrated using a comparable, single Galactic $\alpha_{\mathrm{CO}}$ of $6.5 M_{\odot}\left(\mathrm{K} \mathrm{km} \mathrm{s}^{-1} \mathrm{pc}^{2}\right)^{-1}$ (including the helium contribution) for different types of star-forming galaxies, including SMGs.

We also note that only two of our target sources, AzTEC/C5 and $\mathrm{C} 17$, have $\mathrm{CO}$-inferred $M_{\text {gas }}$ estimates available, and when the different assumptions about $\alpha_{\mathrm{CO}}$ are taken into account, they agree within a factor of two with our dust-based values (being either lower or higher; we refer to M17b, and references therein).

Finally, because the source sizes we derived refer to the FWHM extent, the surface densities were calculated as $\Sigma_{\mathrm{SFR}}=$ $S F R /\left(2 \pi R^{2}\right)$ and $\Sigma_{\text {gas }}=M_{\text {gas }} /\left(2 \pi R^{2}\right)$. The associated uncertainties were propagated from the uncertainties in SFR, $M_{\text {gas }}$, and size.

The K-S diagram of our SMGs is shown in the left panel in Fig. 1, while our data are compared with literature studies in the right panel of the figure. The individual sources are colourcoded according to the distance from the main sequence (MS) as defined by Speagle et al. (2014). We also show the binned version of the data, where the sample was divided into MS objects and super-MS objects or starbursts (defined to be offset from the MS mid-line by a factor of $>3$; see M17b). The linear least squares fits $\left(\log \Sigma_{\mathrm{SFR}}=a \times \log \Sigma_{\text {gas }}+b\right)$ through the binned data points yielded the slope and $y$-intercept of ( $a=0.81 \pm 0.01, b=-1.89 \pm 0.05)$ for the MS SMGs, and $(a=0.84 \pm 0.39, b=-1.81 \pm 1.84)$ for the starburst SMGs. The quoted uncertainties in the fit parameters represent the $1 \sigma$ standard deviation errors, and they were derived from the $\Sigma_{\mathrm{SFR}}$ uncertainties. As illustrated in Fig. 1, our SMG $\Sigma_{\mathrm{SFR}}-\Sigma_{\text {gas }}$ relations have flatter slopes and higher zero points than the K98 relation and the D10 relationships for normal disks and starbursts (with different $\alpha_{\mathrm{CO}}$ values), where the former is very similar to the canonical K98 relation. However, our SMGs have extreme gas surface densities of $\Sigma_{\text {gas }} \gtrsim 10^{3.9} M_{\odot} \mathrm{pc}^{-2}$ on average, and hence we are mostly probing a different $\Sigma_{\text {gas }}$ regime than K98 and
D10 (but using dust rather than CO to estimate $M_{\text {gas }}$ ). Such high densities make the gaseous interstellar medium (ISM) highly optically thick even in the re-radiated IR, and the radiation pressure on dust grains makes the system become Eddington-limited (e.g. Ballantyne et al. 2013; Thompson \& Krumholz 2016, and references therein). Interestingly, the K-S slope for the radiationpressure-supported, Eddington-limited disk is expected to be unity (the stellar radiative flux $F_{\star} \propto \Sigma_{\mathrm{SFR}}$, and the Eddington flux $F_{\text {Edd }} \propto \Sigma_{\text {gas }}$; Thompson et al. 2005; Ostriker \& Shetty 2011), which is broadly consistent with our results, particularly for starburst SMGs for which the K-S slope is consistent with unity within $\sim 0.4 \sigma$.

Our best-fit scaling relations shown in Fig. 1 suggest that the SFE is fairly weakly dependent on $\Sigma_{\text {gas }}$ at the high densities probed (SFE $=\Sigma_{\mathrm{SFR}} / \Sigma_{\text {gas }} \propto \Sigma_{\text {gas }}^{-0.19 \pm 0.01}$ for the MS SMGs, and SFE $\propto \Sigma_{\text {gas }}^{-0.16 \pm 0.39}$ above the MS). To estimate the global SFEs of our SMGs, we fit the binned data with slopes constrained to unity. On average, our MS SMGs are consistent with a constant global SFE of $21_{-1}^{+2} \%$ per $100 \mathrm{Myr}$, while that for our starburst SMGs is $27_{-6}^{+6} \%$ per 100 Myr. The corresponding gas depletion times are $\tau_{\text {dep }}=\mathrm{SFE}^{-1} \simeq 480_{-45}^{+20} \mathrm{Myr}$ and $\simeq 370_{-67}^{+106} \mathrm{Myr}$, respectively.

If the gas scale heights $\left(h \propto \Sigma_{\text {gas }} / \rho_{\text {gas }}\right.$, where $\rho_{\text {gas }}$ is the gas volume density) do not change much among different sources, the K-S law $\Sigma_{\mathrm{SFR}} \propto \Sigma_{\text {gas }}^{1.4 \pm 0.15}$ (K98) is consistent with $\rho_{\mathrm{SFR}} \propto \rho_{\text {gas }} / \tau_{\mathrm{ff}} \propto \rho_{\text {gas }}^{1.5}$, where $\tau_{\mathrm{ff}} \propto \rho_{\text {gas }}^{-0.5}$ is the free-fall timescale. Hence, a possible interpretation is that the $\mathrm{K}-\mathrm{S}$ relation is a manifestation of star formation being predominantly driven by large-scale gravitational disk instabilities with a characteristic dynamical (fragmentation) timescale given by that of free-fall collapse (e.g. Kennicutt 1989; Elmegreen 2002). The K-S relations and $\tau_{\text {dep }}\left(\Sigma_{\text {gas }}\right)$ dependencies we derived are shallower than what would be expected from this free-fall 
paradigm, which could reflect the fact that our measurements are averaged over entire SMGs, and are hence expected to be sensitive to fairly similar ISM characteristics across the sample (e.g. Krumholz \& Thompson 2007; Bigiel et al. 2008).

There are a number of critical assumptions (e.g. $\alpha_{\mathrm{CO}}$ ) and caveats in the above analysis. For example, a lower value of $\alpha_{\mathrm{CO}}=0.8 M_{\odot}\left(\mathrm{K} \mathrm{km} \mathrm{s}^{-1} \mathrm{pc}^{2}\right)^{-1}$, which is often adopted for ultraluminous infrared galaxies (ULIRGs; Downes \& Solomon 1998), might be more appropriate for SMGs than a Galactic value. In Fig. B.1, we show two alternative K-S diagrams, one derived by assuming the aforementioned ULIRG $\alpha_{\mathrm{CO}}$ factor for all of our sources, and another one with a bimodal $\alpha_{\mathrm{CO}}$ distribution, namely a ULIRG-like value for the starburst SMGs, and the same Galactic value for the MS objects as in Fig. 1. We stress that these different assumptions about the $\alpha_{\mathrm{CO}}$ value do not influence the K-S slope values quoted above, only the normalisations (see Appendix B).

Another caveat is that the dust-emitting sizes of SMGs are found to be more compact that the spatial extent of their molecular gas reservoir (see M17a, and references therein), and hence our $\Sigma_{\text {gas }}$ values could well be overestimated. On the other hand, M17a found that the observed-frame $3 \mathrm{GHz}$ radio-emitting sizes of the target SMGs (see Fig. A.1) have a median value comparable to that of the CO-emitting gas component measured through mid- $J$ rotational transitions by Tacconi et al. (2006) for their sample of SMGs (consistent with the SMGs' $1.4 \mathrm{GHz}$ and $\mathrm{CO}$ sizes studied by Bothwell et al. 2010). Hence, one might think that the extent of radio emission is a better estimate of the distribution of molecular gas than the rest-frame far-IR emission. However, it should be noted that the molecular gas reservoir of SMGs probed through $\mathrm{CO}(J=1-0)$ observations is found to be more extended than the denser and warmer component giving rise to mid- $J$ CO emission (we refer to the discussion in M17a). Nevertheless, we also derived the $\Sigma_{\text {gas }}$ values using the radio sizes from M17a, and constructed another version of the K-S diagram, which is shown in the top panel in Fig. C.1 (the bottom panel has also $\Sigma_{\text {SFR }}$ calculated over the $3 \mathrm{GHz}$ size). In this case, we derived a highly sublinear $(a=0.40 \pm 0.07)$ and even negative slope ( $a=-0.16 \pm 0.02)$ for the MS and super-MS objects, which suggests that the $3 \mathrm{GHz}$ sizes are not universally representative of our SMGs' molecular gas extent. This raises the question of which size scale is the most appropriate to compute $\Sigma_{\text {gas }}$, and if the $\mathrm{CO}$ emission size is used, then which $\mathrm{CO}$ transition is the most relevant: $J=1-0$ to probe the full, diffuse molecular gas component, or a higher $J$ transition, which arises from a denser and warmer gas associated with an on-going star formation. Our results are in line with K98, who suggested that it is vital to correlate the values of $\Sigma_{\mathrm{SFR}}$ and $\Sigma_{\text {gas }}$ over regions co-equal in size.

\section{Summary and conclusions}

We used ALMA to carry out a 0'.2 resolution, $870 \mu \mathrm{m}$ continuum imaging survey of a sample of SMGs in COSMOS. When combined with the source size information provided by these observations, our previous dust-based SFR and gas mass estimates for these sources allowed us to examine their K-S type, $\Sigma_{\mathrm{SFR}}-\Sigma_{\text {gas }}$ scaling law. The dust-inferred $M_{\text {gas }}$ values used in the analysis are based on the critical assumption of a uniform Galactic CO-to- $\mathrm{H}_{2}$ conversion factor. We found that the average relationships for our MS and starburst SMGs are
$\Sigma_{\mathrm{SFR}} \propto \Sigma_{\text {gas }}^{0.81 \pm 0.01}$ and $\Sigma_{\mathrm{SFR}} \propto \Sigma_{\text {gas }}^{0.84 \pm 0.39}$. The MS SMGs are consistent with an average constant global SFE of about $21 \%$ per $100 \mathrm{Myr}$, while that of starburst SMGs is somewhat higher, about 27\% per 100 Myr. These SFEs correspond to gas consumption times of $\sim 480 \mathrm{Myr}$ and $370 \mathrm{Myr}$, respectively. The gas surface densities of the studied SMGs are typically $\Sigma_{\text {gas }} \gtrsim 10^{3.9} M_{\odot} \mathrm{pc}^{-2}$, which suggest that the sources exceed the Eddington limit from radiation pressure on dust. Moreover, the slightly sub-linear, or quasi-linear $\Sigma_{\mathrm{SFR}}-\Sigma_{\text {gas }}$ relations we derived are in broad agreement with the theoretical expectation of the SFR and gas surface densities being linearly correlated with each other for the radiation pressure supported, Eddington-limited disk. Our study also demonstrates how the source size can be one of the major bottlenecks in deriving the K-S law of SMGs, and this warrants further observations of the gas distribution in these galaxies.

Acknowledgements. We thank our referee for constructive comments and useful suggestions that helped us improve this paper. We also thank Dirk Petry for his help with our ALMA Cycle 4 data products. This research was funded by the European Union's Seventh Framework programme under grant agreement 337595 (ERC Starting Grant, "CoSMass"). M.A. acknowledges partial support from FONDE-CYT through grant 1140099. A.K. acknowledges support by the Collaborative Research Council 956, sub-project A1, funded by the Deutsche Forschungsgemeinschaft (DFG). This paper makes use of the following ALMA data: ADS/JAO.ALMA\#2016.1.00478.S. ALMA is a partnership of ESO (representing its member states), NSF (USA) and NINS (Japan), together with NRC (Canada), NSC and ASIAA (Taiwan), and KASI (Republic of Korea), in cooperation with the Republic of Chile. The Joint ALMA Observatory is operated by ESO, AUI/NRAO and NAOJ.

\section{References}

Aretxaga, I., Wilson, G. W., Aguilar, E., et al. 2011, MNRAS, 415, 3831 Ballantyne, D. R., Armour, J. N., \& Indergaard, J. 2013, ApJ, 765, 138 Bigiel, F., Leroy, A., Walter, F., et al. 2008, AJ, 136, 2846

Bothwell, M. S., Chapman, S. C., Tacconi, L., et al. 2010, MNRAS, 405, 219 Bouché, N., Cresci, G., Davies, R., et al. 2007, ApJ, 671, 303 Brisbin, D., et al. 2017, A\&A, submitted

Casey, C. M., Narayanan, D., \& Cooray, A. 2014, Phys. Rep., 541, 45 da Cunha, E., Walter, F., Smail, I., et al. 2015, ApJ, 806, 110 Daddi, E., Bournaud, F., Walter, F., et al. 2010a, ApJ, 713, 686 Daddi, E., Elbaz, D., Walter, F., et al. 2010b, ApJ, 714, L118 (D10b) Downes, D., \& Solomon, P. M. 1998, ApJ, 507, 615

Elmegreen, B. G. 2002, ApJ, 577, 206

García-Burillo, S., Usero, A., Alonso-Herrero, A., et al. 2012, A\&A, 539, A8 Genzel, R., Tacconi, L. J., Gracia-Carpio, J., et al. 2010, MNRAS, 407, 2091 Genzel, R., Tacconi, L. J., Lutz, D., et al. 2015, ApJ, 800, 20

Hodge, J. A., Riechers, D., Decarli, R., et al. 2015, ApJ, 798, L18

Hodge, J. A., Swinbank, A. M., Simpson, J. M., et al. 2016, ApJ, 833, 103

Kennicutt, R. C., Jr. 1989, ApJ, 344, 685

Kennicutt, R. C., Jr. 1998, ApJ, 498, 541 (K98)

Kennicutt, R. C., \& Evans, N. J. 2012, ARA\&A, 50, 531

Krumholz, M. R., \& Thompson, T. A. 2007, ApJ, 669, 289

Liu, G., Koda, J., Calzetti, D., Fukuhara, M., \& Momose, R. 2011, ApJ, 735, 63 McMullin, J. P., Waters, B., Schiebel, D., et al. 2007, Astronomical Data Analysis Software and Systems XVI, ASP Conf. Ser., 376, 127

Miettinen, O., Novak, M., Smolčić, V., et al. 2017a, A\&A, 602, A54 (M17a)

Miettinen, O., Delvecchio, I., Smolčić, V., et al. 2017b, A\&A, submitted (M17b) Momose, R., Koda, J., Kennicutt, R. C., Jr., et al. 2013, ApJ, 772, L13 Ostriker, E. C., \& Shetty, R. 2011, ApJ, 731, 41

Schmidt, M. 1959, ApJ, 129, 243

Scoville, N., Sheth, K., Aussel, H., et al. 2016, ApJ, 820, 83

Simpson, J. M., Smail, I., Swinbank, A. M., et al. 2015, ApJ, 799, 81

Speagle, J. S., Steinhardt, C. L., Capak, P. L., \& Silverman, J. D. 2014, ApJS, 214,15

Tacconi, L. J., Neri, R., Chapman, S. C., et al. 2006, ApJ, 640, 228

Tacconi, L. J., Genzel, R., Neri, R., et al. 2010, Nature, 463, 781

Thompson, T. A., Quataert, E., \& Murray, N. 2005, ApJ, 630, 167

Thompson, T. A., \& Krumholz, M. R. 2016, MNRAS, 455, 334

Wyder, T. K., Martin, D. C., Barlow, T. A., et al. 2009, ApJ, 696, 1834 
Appendix A: ALMA $870 \mu \mathrm{m}$ images, the dust-emitting sizes, and the average gas and SFR surface densities

The ALMA $870 \mu \mathrm{m}$ images towards AzTEC/C1-C27 are shown in Fig. A.1, and the derived source sizes are tabulated in Table A.1. In Table A.2, we list the values of the binned average data points $\left(\Sigma_{\text {gas }}\right.$ and $\left.\Sigma_{\text {SFR }}\right)$ plotted in Fig. 1.

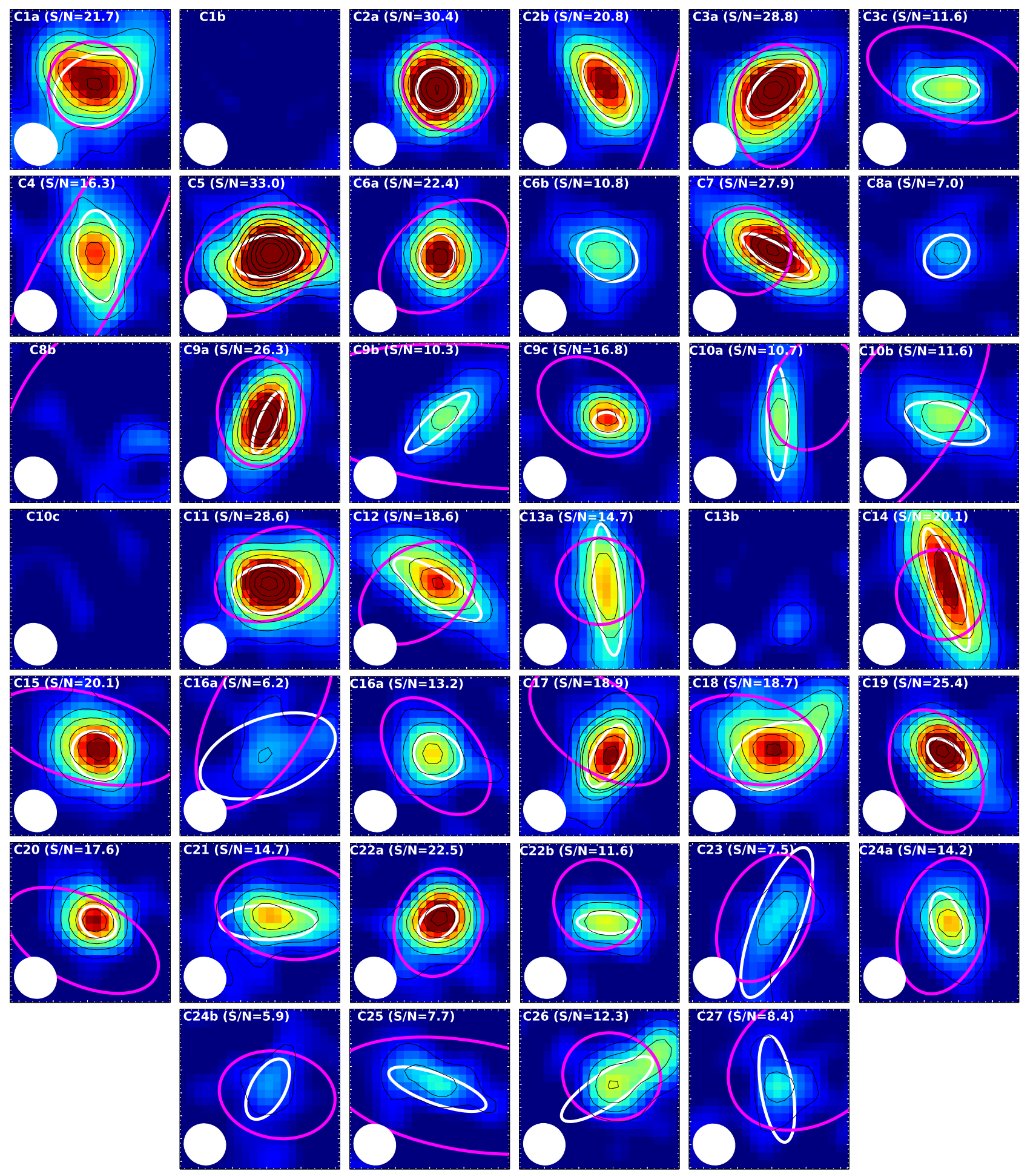

Fig. A.1. Observed-frame $870 \mu \mathrm{m}$ ALMA images towards AzTEC/C1-C27. Each image is centred on the ALMA $870 \mu \mathrm{m}$ peak position (except the non-detections (AzTEC/C1b, C8b, C10c, and C13b), which are centred on the ALMA $1.3 \mathrm{~mm}$ position), is 0 !'7 $\times 0$ "!7 in size, oriented such that north is up and east is left, and displayed in a common linear colour-scale. The contour levels start from $3 \sigma$, and progress in steps of $3 \sigma$. The detection $S / N_{870 \mu \mathrm{m}}$ ratio is indicated in parenthesis. The white and magenta ellipses show the deconvolved FWHM source sizes at $870 \mu \mathrm{m}$ and $3 \mathrm{GHz}$ (the present study and M17a, respectively). The ALMA synthesised beam FWHM is shown in the bottom left of each panel. 
Table A.1. Source sample and the sizes derived through Gaussian fits.

\begin{tabular}{|c|c|c|c|}
\hline Source ID & $z$ & $\begin{array}{c}F W H M_{\text {maj }} \times F W H M_{\min }{ }^{a} \\
{\left[{ }^{\prime \prime}\right]}\end{array}$ & $\begin{array}{r}\mathrm{PA}^{b} \\
{\left[{ }^{\circ}\right]}\end{array}$ \\
\hline AzTEC/C1a & $4.7^{c}$ & $0.40_{-0.05}^{+0.04} \times 0.31_{-0.04}^{+0.04}$ & $113.7_{-21.1}^{+19.2}$ \\
\hline $\mathrm{AzTEC} / \mathrm{C} 2 \mathrm{a}$ & $3.179^{c}$ & $0.19_{-0.04}^{+0.03} \times 0.18_{-0.04}^{+0.03}$ & $166.4_{-40.0}^{+35.5}$ \\
\hline AzTEC/C2b & $1.10_{-1.10}^{+2.60}$ & $0.32_{-0.03}^{+0.04} \times 0.16_{-0.03}^{+0.03}$ & $28.4_{-7.5}^{+8.6}$ \\
\hline $\mathrm{AzTEC} / \mathrm{C} 3 \mathrm{a}^{d}$ & $1.125^{c}$ & $0.32_{-0.02}^{+0.02} \times 0.16_{-0.02}^{+0.02}$ & $132.9_{-5.5}^{+5.7}$ \\
\hline $\mathrm{AzTEC} / \mathrm{C} 3 \mathrm{c}^{d}$ & $2.03_{-0.31}^{+1.19}$ & $0.29_{-0.06}^{+0.05} \times 0.12_{-0.10}^{+0.05}$ & $88.1_{-17.0}^{+13.7}$ \\
\hline AzTEC/C4 & $5.30_{-1.10}^{+0.70}$ & $0.40_{-0.05}^{+0.05} \times 0.19_{-0.04}^{+0.04}$ & $4.0_{-8.2}^{+8.7}$ \\
\hline AzTEC/C5 & $4.3415^{c}$ & $0.31_{-0.03}^{+0.02} \times 0.18_{-0.03}^{+0.02}$ & $99.4_{-7.4}^{+7.1}$ \\
\hline AzTEC/C6a & $2.494^{c}$ & $0.20_{-0.04}^{+0.03} \times 0.15_{-0.04}^{+0.03}$ & $171.4_{-30.8}^{+39.1}$ \\
\hline AzTEC/C6b & $2.513^{c}$ & $0.27_{-0.08}^{+0.07} \times 0.21_{-0.08}^{+0.07}$ & $65.9_{-38.2}^{+44.4}$ \\
\hline AzTEC/C7 & $3.06_{-1.76}^{+1.88}$ & $0.35_{-0.02}^{+0.03} \times 0.10_{-0.03}^{+0.02}$ & $59.6_{-3.7}^{+3.5}$ \\
\hline AzTEC/C8a & $3.62^{c}$ & $0.21_{-0.13}^{+0.07} \times 0.17_{-0.13}^{+0.11}$ & $127.0_{-42.9}^{+36.8}$ \\
\hline AzTEC/C9a & $2.68_{-0.51}^{+0.24}$ & $0.29_{-0.03}^{+0.02} \times 0.08_{-0.06}^{+0.03}$ & $157.0_{-4.9}^{+4.7}$ \\
\hline AzTEC/C9b & $2.8837^{c}$ & $0.37_{-0.08}^{+0.06} \times<0.09$ & $132.9_{-7.3}^{+7.7}$ \\
\hline $\mathrm{AzTEC} / \mathrm{C} 9 \mathrm{c}$ & $2.9219^{c}$ & $0.12_{-0.05}^{+0.05} \times<0.09$ & $85.6_{-43.8}^{+24.0}$ \\
\hline AzTEC/C10a & $3.40_{-0.59}^{+3.60}$ & $0.50_{-0.09}^{+0.08} \times<0.09$ & $0.4_{-5.3}^{+5.5}$ \\
\hline AzTEC/C10b & $2.90_{-0.90}^{+0.30}$ & $0.38_{-0.07}^{+0.07} \times 0.16_{-0.06}^{+0.05}$ & $73.6_{-11.9}^{+10.4}$ \\
\hline $\mathrm{AzTEC} / \mathrm{C} 11^{d}$ & $4.30_{-3.33}^{+0.07}$ & $0.31_{-0.03}^{+0.02} \times 0.21_{-0.03}^{+0.02}$ & $95.5_{-12.4}^{+10.9}$ \\
\hline AzTEC/C12 & $3.25_{-0.51}^{+0.16}$ & $0.45_{-0.05}^{+0.05} \times 0.14_{-0.03}^{+0.04}$ & $56.3_{-4.7}^{+4.6}$ \\
\hline AzTEC/C13a & $2.01_{-0.49}^{+0.15}$ & $0.58_{-0.07}^{+0.07} \times 0.11_{-0.07}^{+0.04}$ & $6.0_{-3.6}^{+3.7}$ \\
\hline AzTEC/C14 & $4.58_{-0.68}^{+0.25}$ & $0.62_{-0.06}^{+0.05} \times 0.11_{-0.04}^{+0.03}$ & $18.1_{-2.3}^{+2.4}$ \\
\hline AzTEC/C15 & $3.91_{-2.35}^{+0.28}$ & $0.24_{-0.04}^{+0.03} \times 0.20_{-0.03}^{+0.04}$ & $53.2_{-44.9}^{+40.2}$ \\
\hline AzTEC/C16a & $3.15_{-1.54}^{+0.62}$ & $0.62_{-0.19}^{+0.18} \times 0.33_{-0.13}^{+0.12}$ & $110.4_{-20.5}^{+28.3}$ \\
\hline AzTEC/C16b & $2.39_{-0.56}^{+0.27}$ & $0.22_{-0.09}^{+0.04} \times 0.19_{-0.04}^{+0.08}$ & $55.1_{-42.8}^{+44.5}$ \\
\hline AzTEC/C17 & $4.542^{c}$ & $0.30_{-0.04}^{+0.03} \times 0.12_{-0.05}^{+0.03}$ & $153.1_{-7.4}^{+7.2}$ \\
\hline AzTEC/C18 & $3.15_{-0.44}^{+0.13}$ & $0.43_{-0.05}^{+0.05} \times 0.27_{-0.04}^{+0.04}$ & $112.6_{-11.4}^{+11.2}$ \\
\hline AzTEC/C19 & $2.87_{-0.41}^{+0.11}$ & $0.20_{-0.02}^{+0.03} \times 0.11_{-0.02}^{+0.03}$ & $51.2_{-11.7}^{+12.9}$ \\
\hline AzTEC/C20 & $3.06_{-0.54}^{+0.13}$ & $0.17_{-0.05}^{+0.03} \times 0.13_{-0.05}^{+0.03}$ & $56.5_{-41.7}^{+43.2}$ \\
\hline AzTEC/C21 & $2.70_{-0.40}^{+1.30}$ & $0.42_{-0.06}^{+0.06} \times 0.15_{-0.06}^{+0.04}$ & $89.9_{-7.0}^{+6.7}$ \\
\hline AzTEC/C22a & $1.599^{c}$ & $0.19_{-0.02}^{+0.03} \times 0.13_{-0.03}^{+0.03}$ & $127.8_{-15.6}^{+18.4}$ \\
\hline AzTEC/C22b & $1.599^{c}$ & $0.27_{-0.06}^{+0.05} \times<0.09$ & $87.4_{-12.8}^{+11.3}$ \\
\hline AzTEC/C23 & $2.10_{-0.41}^{+0.46}$ & $0.70_{-0.19}^{+0.18} \times 0.20_{-0.11}^{+0.09}$ & $158.7_{-9.0}^{+9.1}$ \\
\hline AzTEC/C24a & $2.01_{-0.46}^{+0.19}$ & $0.27_{-0.05}^{+0.05} \times 0.13_{-0.05}^{+0.05}$ & $16.5_{-13.7}^{+16.2}$ \\
\hline $\mathrm{AzTEC} / \mathrm{C} 24 \mathrm{~b}^{d}$ & $2.10_{-0.63}^{+0.08}$ & $0.29_{-0.13}^{+0.10} \times 0.15_{-0.15}^{+0.10}$ & $151.1_{-34.9}^{+33.2}$ \\
\hline AzTEC/C25 & $2.51^{c}$ & $0.45_{-0.12}^{+0.11} \times 0.13_{-0.13}^{+0.07}$ & $70.3_{-11.0}^{+10.5}$ \\
\hline AzTEC/C26 & $5.06_{-0.90}^{+0.08}$ & $0.47_{-0.07}^{+0.07} \times 0.14_{-0.07}^{+0.04}$ & $123.4_{-6.2}^{+6.2}$ \\
\hline AzTEC/C27 & $2.77_{-0.47}^{+0.88}$ & $0.31_{-0.11}^{+0.10} \times 0.20_{-0.12}^{+0.08}$ & $9.1_{-17.9}^{+40.1}$ \\
\hline
\end{tabular}

Notes. The sources AzTEC/C1b, C8b, C10c, and C13b were not detected in our ALMA $870 \mu$ m survey, while AzTEC/C3b was not covered by our ALMA pointings (see Sect. 2). ${ }^{(a)}$ Deconvolved FWHM of the major and minor axes derived through Gaussian fits in the image plane using the AIPS task JMFIT. ${ }^{(b)}$ Major axis position angle of the fitted Gaussian measured from north through east. Formally, the PA lies in the range $\mathrm{PA} \in\left[0^{\circ}, 180^{\circ}\right]$, but some of the tabulated values have uncertainties that place the PA value being outside this range. However, the PA is symmetrical under a $180^{\circ}$ rotation. ${ }^{(c)}$ Spectroscopic redshift (see Brisbin et al. 2017, and references therein). ${ }^{(d)}$ No MAGPHYS SED could be derived for the source, while AzTEC/C11 and C24b were excluded from the SED analysis because they are likely to host an active nucleus (M17b).
Table A.2. Molecular gas and SFR surface densities of the binned average data points shown in Fig. 1.

\begin{tabular}{cc}
\hline \hline $\log \left(\Sigma_{\mathrm{gas}} / M_{\odot} \mathrm{pc}^{-2}\right)^{a}$ & $\log \left(\Sigma_{\mathrm{SFR}} / M_{\odot} \mathrm{yr}^{-1} \mathrm{kpc}^{-2}\right)$ \\
\hline \multicolumn{2}{c}{ MS objects $^{b}$} \\
\hline $3.89 \pm 0.14$ & $1.26 \pm 0.07$ \\
$4.30 \pm 0.05$ & $1.60 \pm 0.13$ \\
$4.63 \pm 0.04$ & $1.86 \pm 0.16$ \\
$4.88 \pm 0.05$ & $2.03 \pm 0.34$ \\
\hline \multicolumn{2}{c}{ Starbursts $^{c}$} \\
\hline $4.14 \pm 0.06$ & $1.64 \pm 0.26$ \\
$4.43 \pm 0.05$ & $2.03 \pm 0.20$ \\
$4.84 \pm 0.05$ & $2.09 \pm 0.13$ \\
$5.03 \pm 0.07$ & $2.64 \pm 0.20$ \\
\hline
\end{tabular}

Notes. ${ }^{(a)}$ The gas masses used to derive these gas surface densities were estimated using the Scoville et al. (2016) dust continuum method, which is based on the assumption of a uniform, Galactic $\alpha_{\mathrm{CO}}$ conversion factor. ${ }^{(b)}$ The MS definition was adopted from Speagle et al. (2014). (c) The starbursts were defined as objects that lie above the MS mid-line by a factor of $>3$.

\section{Appendix B: K-S diagrams constructed using different $\mathrm{CO}-\mathrm{to}-\mathrm{H}_{2}$ conversion factors}

In the top panel in Fig. B.1, we show a similar K-S diagram to that in Fig. 1, but where all the $\Sigma_{\text {gas }}$ values were calculated by assuming a ULIRG $\alpha_{\mathrm{CO}}$ factor of $0.8 M_{\odot}\left(\mathrm{K} \mathrm{km} \mathrm{s}^{-1} \mathrm{pc}^{2}\right)^{-1}$. The linear least squares fits through the binned averages yielded the slope and $y$-intercept of ( $a=0.81 \pm 0.01, b=-1.16 \pm 0.04)$ for the MS SMGs, and ( $a=0.84 \pm 0.39, b=-1.04 \pm 1.49)$ for the starburst SMGs. The slopes remain the same as in Fig. 1, but the former (latter) normalisation is higher by a factor of 5.37 (5.89). This makes most of our average starburst data points consistent with the D10b starburst sequence.

The K-S diagram shown in the bottom panel in Fig. B.1 was constructed by assuming the same Galactic $\alpha_{\mathrm{CO}}$ factor for the MS SMGs as in Fig. 1, and the aforementioned ULIRG-like factor for starbursts. This creates a clear bimodal distribution in the K-S plane (starbursts versus MS objects). The corresponding best-fit parameters for the MS SMGs are the same as in Fig. 1 $(a=0.81 \pm 0.01, b=-1.89 \pm 0.05)$, and for the starbursts they are the same as quoted above $(a=0.84 \pm 0.39, b=-1.04 \pm 1.49)$. 

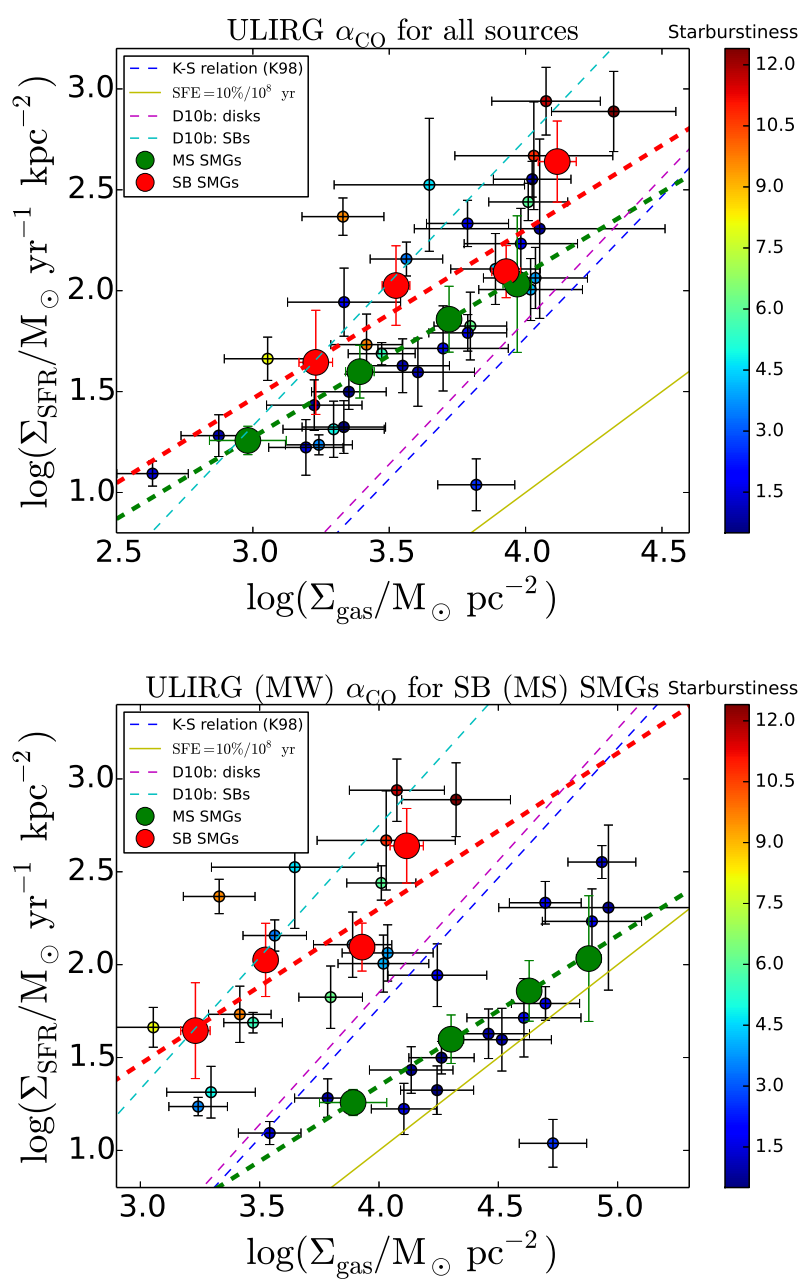

Fig. B.1. Top: similar to Fig. 1, but all the $\Sigma_{\text {gas }}$ values were calculated by scaling the dust-based gas masses by a factor of $0.8 / 6.5$ to make them consistent with a ULIRG $\alpha_{\mathrm{CO}}$ conversion factor of $0.8 M_{\odot}\left(\mathrm{K} \mathrm{km} \mathrm{s}^{-1} \mathrm{pc}^{2}\right)^{-1}$. Bottom: similar to the top panel, but only the starburst SMGs' $\Sigma_{\text {gas }}$ values were calculated by using the aforementioned ULIRG $\alpha_{\text {CO }}$ factor, while a Galactic value was assumed for the MS objects. The plotting ranges of the two panels are different for legibility purposes.

\section{Appendix C: K-S diagrams constructed using the $3 \mathrm{GHz}$ sizes}

In the top panel in Fig. C.1, we show a modified version of Fig. 1 where the gas surface densities were calculated over the $3 \mathrm{GHz}$ radio-emitting sizes (M17a; see the magenta ellipses in Fig. A.1). The K-S diagram shown in the bottom panel in Fig. C.1 has both the SFR and gas surface densities calculated over the $3 \mathrm{GHz}$ sizes. The data were binned separately for the MS and starburst objects, and the three sources that were unresolved at $3 \mathrm{GHz}$ (AzTEC/C1a, C7, and C13a) were incorporated into the binned averages using a right-censored Kaplan-Meier (K-M) survival analysis (see M17a for details). The linear least squares fit parameters were found to be ( $a=0.40 \pm 0.07, b=0.20 \pm 0.24)$ for the MS SMGs, and ( $a=-0.16 \pm 0.02, b=2.74 \pm 0.07)$ for the
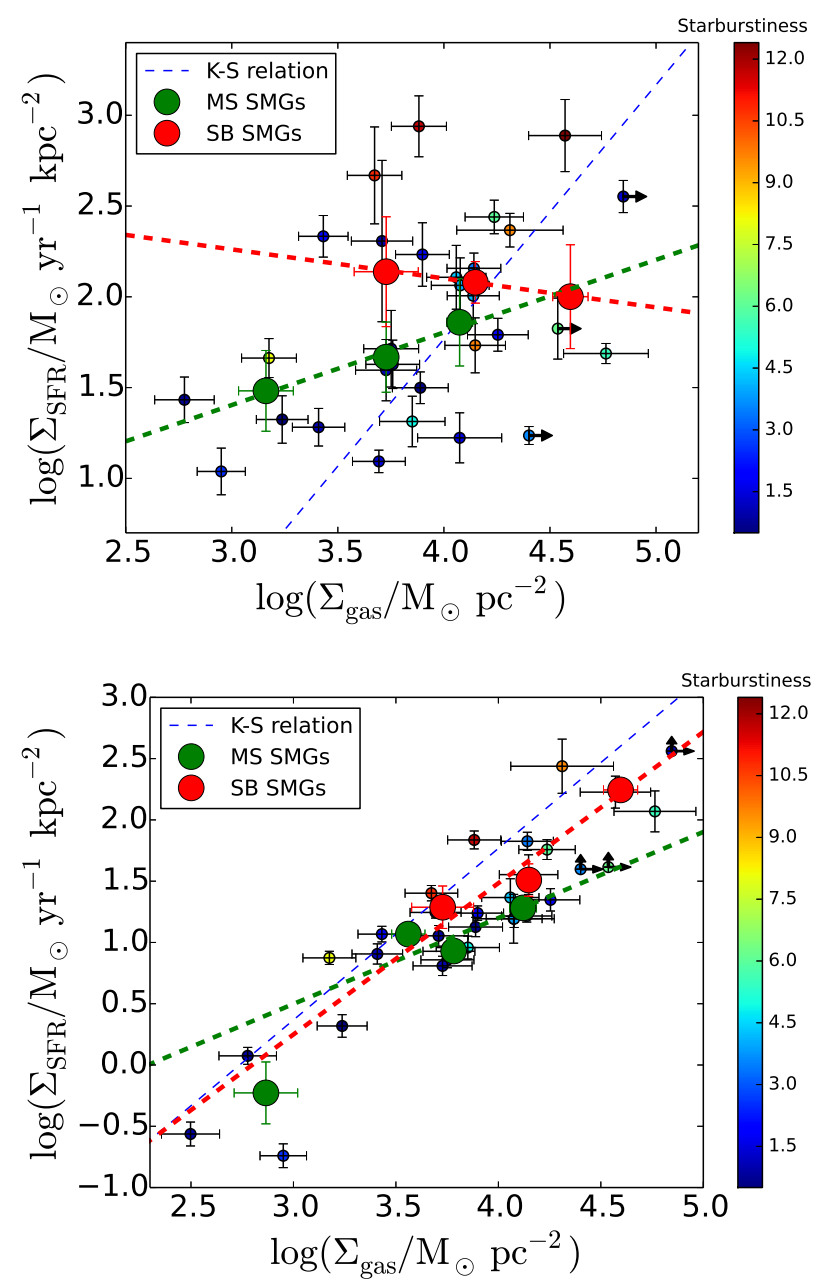

Fig. C.1. Top: similar to Fig. 1, but $\Sigma_{\text {gas }}$ was calculated over the $3 \mathrm{GHz}$ radio sizes derived by M17a (the magenta ellipses in Fig. A.1). Each bin contains five sources. Bottom: similar to the top panel, but both $\Sigma_{\mathrm{gas}}$ and $\Sigma_{\mathrm{SFR}}$ were calculated over the $3 \mathrm{GHz}$ radio sizes. Each MS (SB) bin contains four (five) sources, where the one additional source compared to the top panel is the $3 \mathrm{GHz}$ detected SMG AzTEC/C $8 \mathrm{~b}$. In both panels, the three sources unresolved at $3 \mathrm{GHz}$ (lower limit to $\Sigma_{\text {gas }}$ in the top panel, and to both $\Sigma_{\text {gas }}$ and $\Sigma_{\text {SFR }}$ in the bottom panel) were incorporated into the binned averages using a right-censored K-M survival analysis. The K98 relationship is shown for comparison. The plotting ranges of the two panels are different for legibility purposes.

starbursts in the top panel. The corresponding parameters for the data plotted in the bottom panel are $(a=0.70 \pm 0.30, b=-1.61 \pm$ $1.20)$ and $(a=1.23 \pm 0.29, b=-3.45 \pm 1.29)$, respectively. The results suggest that $\Sigma_{\mathrm{SFR}}$ and $\Sigma_{\text {gas }}$ should be compared over common size scales (K98). However, as discussed in M17a, the $3 \mathrm{GHz}$ radio emission might not always be probing the spatial extent of active high-mass star formation (and hence $\Sigma_{\mathrm{SFR}}$ ), but instead the radio-emitting region can be puffed up as a result of the same galaxy interaction that triggers the SMG phase. Hence, in the main text we focused on the K-S relation derived using the $870 \mu \mathrm{m}$ dust-emitting sizes. 